\title{
Expression and clinical significance of negative costimulatory molecules B7-H1, B7-H3 and B7-H4 in the process of colorectal cancer's evolution
}

\author{
Zhichao Qiu $^{1,2}$, Jingyi Wu ${ }^{1,2}$, Yan Wang ${ }^{1,2}$, Fang Wang ${ }^{1,2}$, Xiaosong Ge ${ }^{1}$, Xingxiang Liu ${ }^{3}$, Xiaowei $\mathbf{Q i}^{1}$, \\ Dong Hua ${ }^{1}$, Yong Mao ${ }^{1}$ \\ ${ }^{1}$ Department of Oncology, Affiliated Hospital of Jiangnan University, Wuxi 214062, China; ${ }^{2}$ Department of Public Health and Preventive Medicine, School \\ of Medicine Jiangnan University, Wuxi 214122, China; ${ }^{3}$ Department of Oncology, Second People's Hospital of Taizhou City, Taizhou 317200 , China \\ Contributions: (I) Conception and design: Y Mao, Z Qiu; (II) Administrative support: Y Mao, D Hua; (III) Provision of study materials or patients: \\ X Ge, X Qi, X Liu; (IV) Collection and assembly of data: J Wu, Y Wang, F Wang; (V) Data analysis and interpretation: Z Qiu, F Wang; (VI) \\ Manuscript writing: All authors; (VII) Final approval of manuscript: All authors. \\ Correspondence to: Yong Mao; Dong Hua. Department of Oncology, Affiliated Hospital of Jiangnan University, 200 Huihe Road, Wuxi 214062, \\ China. Email: mydoctorwx@aliyun.com; wx89211@163.com.
}

Background: We made investigations on the expression of negative costimulatory molecules B7-H1, B7-H3, B7-H4 in lesions at various stages of human colorectal cancer (CRC) evolution. We also analyzed the relevance between its expression in CRC and pathological factors in clinic and patient survival time. We finally made a result that we can ensure the clinical significance of B7-H3, B7-H4 expression and the relationship between the $\mathrm{B} 7$ family molecules expression.

Methods: All stages of CRC were collected, including polyps, adenomas, high-grade neoplasms, and colorectal carcinomas. There were 98 cases of resected CRC tissue, 30 cases of polyps, 30 cases of adenomas, and 25 cases of high-grade neoplasia. Then analysis the expression of three negative costimulatory molecules in all stages of colorectal tissue expression patterns, flow cytometry CD $3^{+} \mathrm{T}$ lymphocytes $\mathrm{B} 7$ family molecules to explore the potential value of their expression.

Results: The expression of B7 family molecules in 30 cases of polyps, 30 cases of adenomas, 25 cases of highgrade neoplasia, and 98 cases of CRC tissues revealed that the B7-H1 and B7-H3 expression quantity was found in polyps, adenomas, high-grade neoplasms, as well as they were highly expressed in cancerous tissues. B7-H4 was only expressed in cancerous tissues. In the TNM stage of tumor progression, three negative costimulatory molecules were mainly expressed in the cytoplasm of tumor cells. In CRC in lymphocytes B7-H4 expression was related to patient age, mucinous adenocarcinoma, and lymph node metastasis. Survival analysis showed in CRC it was statistically significant that that the expression of B7-H3 and the survival rate of patients. We found that $92.9 \%$ of CRC patients expressed B7 family negative costimulatory molecules in varying degrees, and the prognosis and co-expression of B7 family negative co-stimulatory molecules were negatively correlated.

Conclusions: Costimulatory molecules B7-H1, B7-H3 were expressed on the early stage of CRC development. The amount of $\mathrm{CD}^{+} \mathrm{T}$ lymphocyte infiltration in $\mathrm{CRC}$ was positively correlated with the survival of patients. They are the earliest molecules to participate in the progress of CRC developing in B7 family negative molecules. This suggests that B7-H1 and B7-H3 are involved in rectal carcinoma at the junction, as well as it is essential throughout the entire evolutionary process. However, it is in CRC tissues that B7-H4 only get expressed, and different expression patterns may have different clinical significances.

Keywords: B7-H1; B7-H3; B7-H4; CD3 ${ }^{+}$; colorectal carcinoma

Submitted May 28, 2018. Accepted for publication Jul 11, 2018.

doi: $10.21037 /$ tcr.2018.07.15

View this article at: http://dx.doi.org/10.21037/tcr.2018.07.15 


\section{Introduction}

Colorectal cancer (CRC) is a common cancer in humans and ranks fourth in the world for morbidity and mortality (1). In China's economically developed areas, it is closer to that found in Western countries, and these areas are ranked second in malignant tumors. Therefore, exploring a mechanism to prevent and reverse the occurrence of CRC has become a major concern in basic research and clinical research in our country.

Now that the occurrence of CRC showed normal from the organization, through polyps, adenomas, high-grade tumor lesions such as the slow progression of cancer to the long process, involves a number of molecular pathways (2). Costimulatory signals and costimulatory molecules were first proposed and confirmed by Brestcher and Cohn in 1970 based on $\mathrm{T}$ cell activation dual signal theory (3). The regulatory of negative costimulatory molecules, their interactions and signaling play an extremely essential part in very complex immune response. Of the costimulatory molecules, the B7 family is the most important (4). These costimulatory signaling molecules provide positive signals for $\mathrm{T}$ cell growth, differentiation, and cytokine production. They also limit, weaken, or terminate $\mathrm{T}$ cell immune responses by providing negative signals and mediating immune escape by a variety of mechanisms that allow tumor cells to escape an immune attack (5). The current study suggests that B7-H1, B7-H3, B7-H4 which are negative costimulatory molecules belong to the B7 family. They are all expressed and negatively regulate the immune response by restraining $\mathrm{T}$ cell activation and proliferation with tumor cells, which takes an extremely important part in the tumor cell immune escape mechanism (6-8). The expression of these three negative costimulatory molecules proteins can be detected in many human tumor tissues (9). Its proteins are widely expressed in many tumor tissues, including kidney cancer, gastric carcinoma, prostate carcinoma, renal carcinoma, and neuroblastoma, and they are also found in tumor blood vessels (10-12).

The present study suggests that the interaction of PD-L1 with its receptor PD-1, which is expressed on T cells, leads to tumor antigen-specific $\mathrm{T}$ cell apoptosis. This is the main mechanism of B7-H1-mediated tumor immune escape (13). Negative correlation between B7$\mathrm{H} 1$ expression and epithelial cell $\mathrm{CD}^{+} \mathrm{T}$ cell infiltration in ovarian cancer (14). This indicates that B7-H1 is expressed directly in tumor cells, and this has a direct inhibitory effect on the $\mathrm{CD}^{+} \mathrm{T}$ cells of tumor cells (15-17). High expression of B7-H4 can protect epidermal cells from anoikis and promote the malignant transformation of epithelial cells $(18,19)$. Thus, it has an important position in the mechanism of immune escape in malignant tumors.

The development of CRC is a slow and lengthy process. However, B7 family are negative costimulatory molecules. They are found in different stages of tumor microenvironment formation and promote tumorigenesis and progression. It is unclear how they exert their respective immunological negative regulatory effects. Therefore, the study of B7 family molecules in the development of CRC could significantly benefit the early detection and improve the therapeutic effect of colorectal carcinoma.

\section{Methods}

\section{Patients and tissue samples}

Approved by the Ethical Review Committee of the Affiliated Hospital of Jiangnan University, 98 cases of CRC undergoing surgical treatment and pathological diagnosis at the Affiliated Hospital of Jiangnan University from 2006 to 2008 were collected. Into the group of cases, the tissue wax block is well preserved. For each case, all of the original hematoxylin and eosin-stained sections was reviewed by the researchers. The analysis of the clinical and pathological factors included the patient sex, age, tumor size, primary tumor (pT), histological grade, lymph node metastasis ( $\mathrm{pN})$, pathological stage, vascular invasion, nerve infiltration, and lymphatic infiltration. The average follow-up duration was 50.0 months, with a range of $0.8-104.0$ months.

\section{Immunobistochemistry (IHC) assay}

IHC assay was performed according to previously described methods using antibodies for B7-H1 (ab205921, Abcam, Hongkong, China), B7-H3 (ab227679, Abcam, Hongkong, China), and B7-H4 (ab209242, Abcam, Hongkong, China). The analysis was the double-blind method was used to investigate the levels of these three negative costimulatory molecules in the B7 family in the HCC tissues. The proportion of tumor cells was scored using the following rules: zero points for no tumor cell staining; one point for $1-25 \%$ tumor cell coloring; two points for $26-50 \%$ tumor cell coloring; three points for $51-75 \%$ tumor cell coloring; four points for $>75 \%$ tumor cell coloring. Protein expression intensity was scored as follows: zero points for no staining, one point for weak staining (light yellow), two 
points for moderate staining (yellowish brown), or three points for strong staining (brown). The staining index was calculated as the sum of the staining intensity and the proportion of tumor cell scores ratio $(0,1,2,3,4,6,8$, 9 , or 12 points). Cut-off values for B7 family molecules expression were selected based on a measurement of heterogeneity using the log-rank test with respect to the overall survival. If the patients were classified as having high expression of $\mathrm{B} 7-\mathrm{H} 1$, the IHC scores were $>6.25$. Using the same method, if the patients were classified as having high expression of $\mathrm{B} 7-\mathrm{H} 3$, the $\mathrm{IHC}$ scores were $>2.75$, and high expression of $\mathrm{B} 7-\mathrm{H} 4$ was determined by $\mathrm{IHC}$ scores $>5.75$.

\section{Flow cytometry analysis}

To determine TIGIT expression in $\mathrm{CD}^{+} \mathrm{T}$ cells, mononuclear cells of 3 fresh tumor tissues and 3 adjacent normal tissues were incubated with fluorescein isothiocyanate-conjugated antihuman $\mathrm{CD} 3$, allophycocyanin-conjugated antihuman CD3, and either phycoerythrin-conjugated anti-B7-H1 (ab205921, Abcam, Hongkong, China), B7-H3 (ab227679, Abcam, Hongkong, China), or B7-H4 (ab209242, Abcam, Hongkong, China) antibody for $30 \mathrm{~min}$ at room temperature. After two washes with phosphate buffered saline (PBS), the PBMCs were analyzed using a flow cytometer (FACSCanto II; BD Biosciences, San Jose, CA, USA).

\section{Statistical analysis}

The most appropriate cutoff value for B7-H1, B7-H3, and $\mathrm{B} 7-\mathrm{H} 4$ scores were obtained by generating receiver operating characteristics (ROC) curves. The association between B7-H1, B7-H3 and B7-H4 expression and clinicopathological parameters was assessed by Pearson chi square test or Fisher exact test. Kaplan-Meier method was used to determine the possibility of overall survival, and logarithmic rank test was used to analyze the data. ROC curve (AUC) and evaluated multivariate analysis can predict the prognosis of CRC patients. The SPSS software package (version 20, IBM, Chicago, IL, USA) was used for statistical analysis, and $\mathrm{P}$ value $<0.05$ was deemed to significant.

\section{Results}

\section{Expression of B7 family molecules in different stages of CRC progression}

Immunohistochemical analysis showed that B7 family molecules (Figure 1) were expressed in the cytoplasm, cell membrane, as well as nucleus of tumor cells. There was nearly no expression of its found in normal tissues. However, the expression of $\mathrm{B} 7-\mathrm{H} 1$ and $\mathrm{B} 7-\mathrm{H} 3$ was significantly higher in polyps, adenomas, as well as high grade tumors than in benign tissues.

\section{Expression rates of $B 7$ family molecules in different stages of CRC progression}

The B7-H1 expression rates in polyps, adenomas, and high-grade neoplasm tissues were $36.7 \%, 33.3 \%$, and $44.0 \%$, respectively. The B7-H3 expression rates in polyps, adenomas, and high-grade neoplasm tissues were $30.0 \%$, $33.3 \%$, and $48.0 \%$, respectively. Among 98 CRC patients, $45.9 \%$ highly expressed B7-H1, 45.9\% highly expressed B7-H3, and 57.1\% highly expressed B7-H4 (Table 1).

\section{Expression patterns of B7 family in different stages of colorectal carcinoma progression}

The expression of the B7 family negative co-stimulatory molecules in different stages of CRC progression had different subcellular localization patterns: cell membrane expression, cytoplasmic expression, and nuclear expression (Figure $2 A, B, C, D)$. The $\mathrm{B} 7-\mathrm{H} 1, \mathrm{~B} 7-\mathrm{H} 3$ molecules were primarily expressed in the nucleus during the polyp stage. B7-H3 molecules were also expressed in the cytoplasm and the membrane, but B7-H4 molecules were not. The B7-H1, B7-H3 molecules were primarily expressed in the nucleus during the adenoma stage, and they were also expressed in infiltrating lymphocytes. The B7-H4 molecules were not expressed in infiltrating lymphocytes. B7-H1 and B7-H3 were primarily expressed in the cytoplasm and membrane during the high-grade neoplasia stage. B7 family were primarily expressed in the cytoplasm and the cell membrane during the tumor stage; they also had selected expression in the nucleus.

\section{The expression of B 7 family negative costimulatory molecules in the nucleus of CRC}

B7 family molecules were also expressed in the cell membrane and the cytoplasm of colorectal carcinoma. The effect of their expression on tumor cells and microenvironment is not known yet. Therefore, the clinical significance of the expression of B7 family molecules in the nucleus of colorectal carcinoma and the regulation 


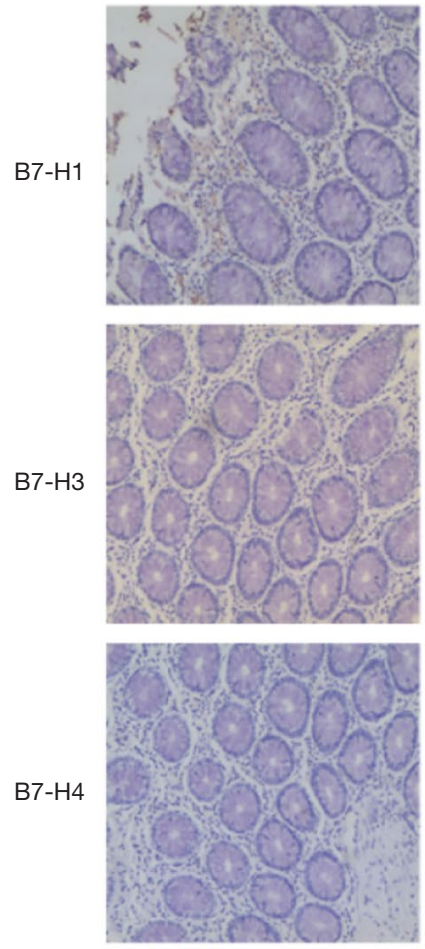

Normal tissue
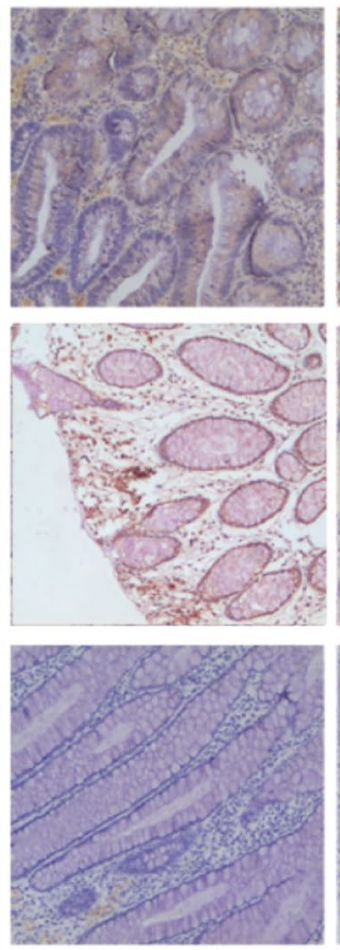

Intestinal polyps
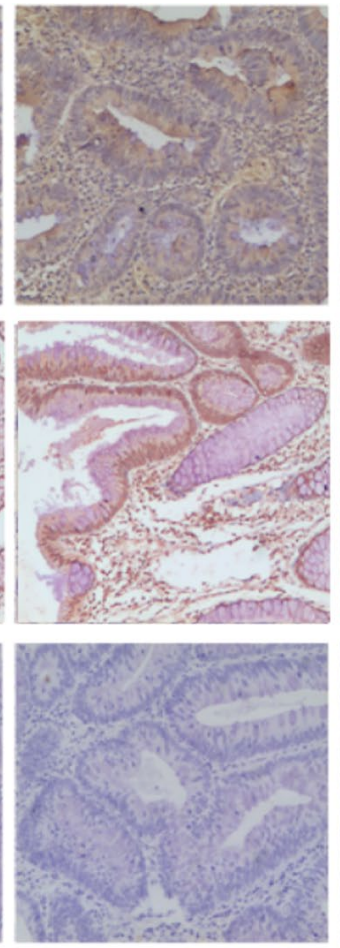

Adenoma
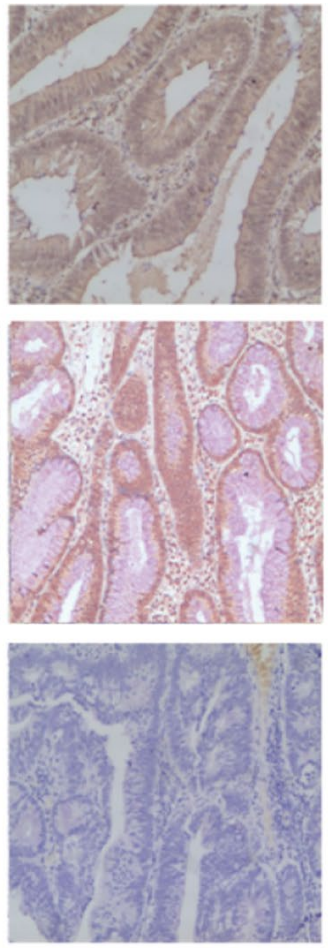

High grade neoplasia
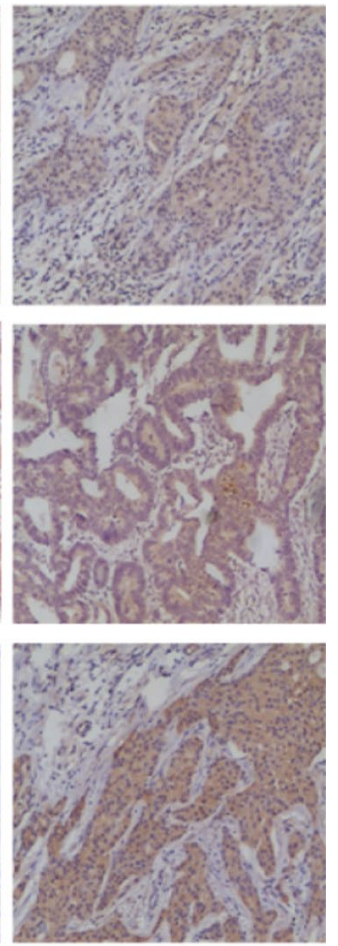

Adenocardinoma

Figure 1 The expression of negative co-stimulatory molecules B7-H1, B7-H3 and B7-H4 in the development of colorectal cancer. B7-H1, B7-H3, and B7-H4 are hardly expressed in normal tissues. With the progression of colorectal cancer, the expression of B7-H1, B7-H3 and B7-H4 gradually increases $(\times 200)$.

Table 1 Expression rates of B7-H1, B7-H3, and B7-H4 molecules in different stages of colorectal cancer progression

\begin{tabular}{lccc}
\hline Pathological stage [n] & B7-H1 (\%) & B7-H3 (\%) & B7-H4 (\%) \\
\hline Intestinal polyps [30] & 36.7 & 30.0 & 0 \\
Adenoma [30] & 33.3 & 33.3 & 0 \\
High grade neoplasia [25] & 44.0 & 48.0 & 0 \\
Adenocarcinoma [98] & 45.9 & 45.9 & 57.1 \\
\hline
\end{tabular}

mechanism of the occurrence and development of colorectal carcinoma will be the focus of our further research direction.

\section{Correlativity between B7-H1 molecule expression and clinic pathological factors in CRC stroma and tumor foci}

According to the expression levels of B7 family molecules, CRC tissues were divided into low expression and high expression (Table 2). Chi-square test statistical analysis showed that the expression level of $\mathrm{B} 7-\mathrm{H} 1$ in interstitial lymphocytes of colorectal carcinoma was unrelated to sex, age, tumor stage, differentiation, tumor location, lymph node metastasis, distant metastasis, Dukes' stage, and whether or not it is a mucinous adenocarcinoma. In cancer foci, the expression level indicated by B7-H1 was unrelated to patient sex, age, distant metastasis, tumor location, tumor stage, lymph node metastasis, Dukes' stage, as well as mucinous adenocarcinoma. It was only related to the degree of differentiation of the patient, which means poor B7-H1 expression may be an independent prognostic factor. Chisquare test statistical analysis indicated that the expression level of B7-H3 molecules in colorectal carcinoma foci was 

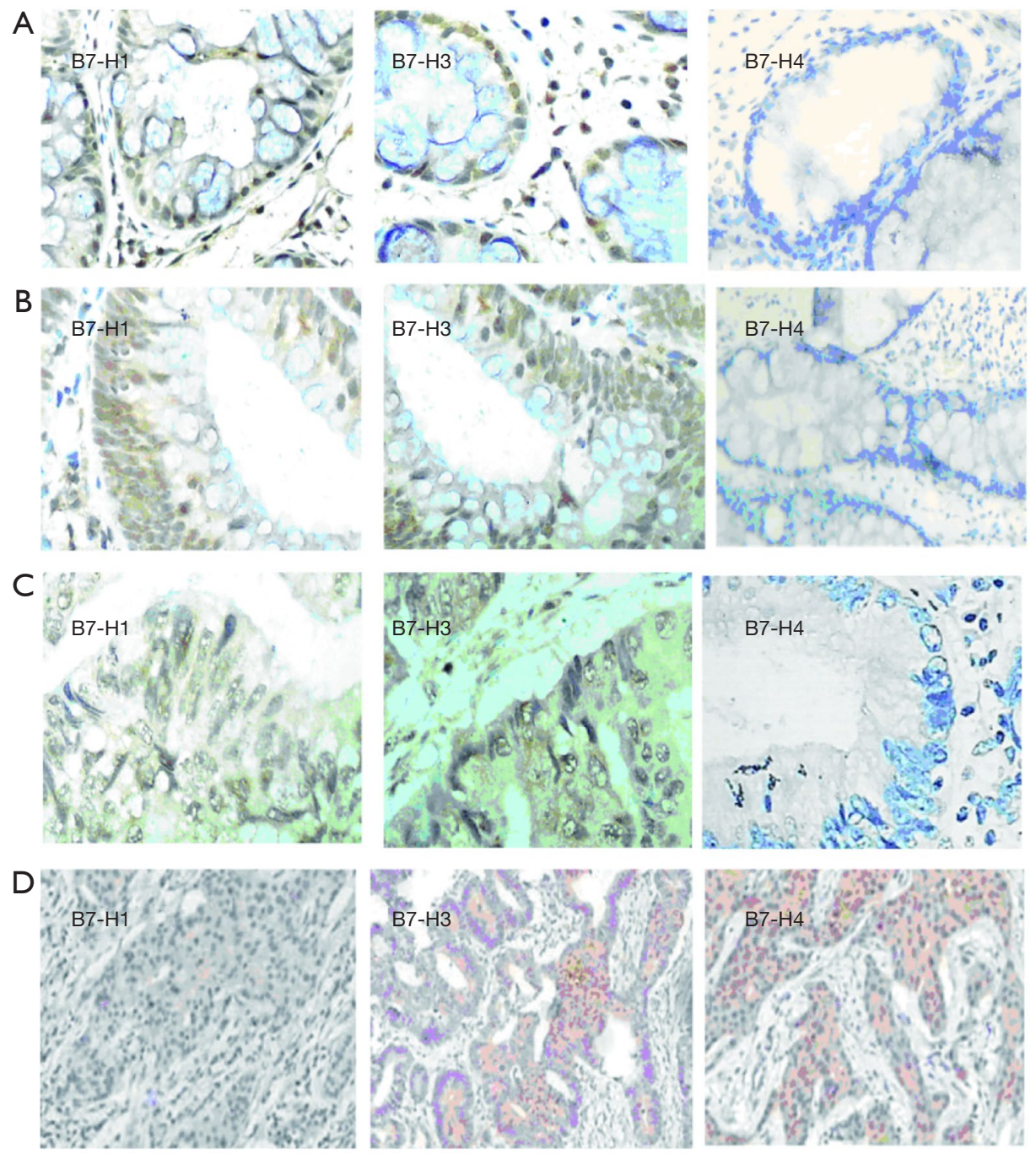

Figure 2 Expression patterns of B7-H1, B7-H3, and B7-H4 in different stages of colorectal cancer progression. In the polyp stage B7-H1 and B7-H3 are mainly nuclear expression (A) (×400). At the stage of adenoma, B7-H1 and B7-H3 mainly expressed in nuclear and infiltrating lymphocytes (B) (x400). In the high-grade neoplastic stage, B7-H1 and B7-H3 are mainly expressed in cell membrane and cytoplasm (C) (×400). At the tumor stage, B7-H1, B7-H3 and B7-H4 mainly expressed in the membrane and cytoplasm (D) ( $\times 200)$.

unrelated to patient sex, age, tumor location, differentiation, tumor stage, lymph node metastasis, distant metastasis, Dukes' stage, as well as mucinous adenocarcinomas. The expression level of $\mathrm{B} 7-\mathrm{H} 3$ in colorectal tissue stromal lymphocytes was unrelated to patient sex, age, tumor location, tumor stage, differentiation, distant metastasis, Dukes' stage, and mucinous adenocarcinoma. Only related to lymph node metastasis; high expression of $\mathrm{B} 7-\mathrm{H} 3$ in colorectal lymphocytes was prone to cause lymph node metastasis. Chi-square test statistical analysis indicated that the expression level of B7-H4 in interstitial lymphocytes of colorectal carcinoma was independent of patient gender, tumor location, tumor stage, differentiation degree, distant metastasis, and Dukes' stage. It was related to patient age, lymph node metastasis, mucinous adenocarcinoma. The expression level of $\mathrm{B} 7-\mathrm{H} 4$ in the foci was unrelated to patient sex, age, differentiation, tumor location, or mucinous adenocarcinoma. However, it was related to tumor stage, distant metastasis, lymph node metastasis, and Dukes' staging. These results suggest that high expression of B7-H4 in lymphocytes and colorectal carcinoma cells is closely correlated with the progression of colorectal 
Table 2 Clinical parameters of B7-H1, B7-H3, and B7-H4

\begin{tabular}{|c|c|c|c|c|c|c|c|c|c|c|}
\hline $\begin{array}{l}\text { Clinical pathological } \\
\text { parameters }\end{array}$ & $\mathrm{n}$ & \multicolumn{3}{|c|}{ Cytoplasm B7-H1 expression } & \multicolumn{3}{|c|}{ Cytoplasm B7-H3 expression } & \multicolumn{3}{|c|}{ Cytoplasm B7-H4 expression } \\
\hline Total & 98 & 53 & 45 & - & 53 & 45 & - & 66 & 32 & - \\
\hline \multicolumn{11}{|l|}{ Gender } \\
\hline Male & 54 & 27 & 27 & 0.419 & 30 & 24 & 0.840 & 34 & 20 & 0.390 \\
\hline \multicolumn{11}{|l|}{ Age } \\
\hline$\geq 60$ & 55 & 27 & 28 & 0.310 & 31 & 24 & 0.690 & 31 & 24 & $0.020^{*}$ \\
\hline$<60$ & 43 & 26 & 17 & & 22 & 21 & & 35 & 8 & \\
\hline \multicolumn{11}{|l|}{ Location } \\
\hline \multicolumn{11}{|l|}{ Differentiation } \\
\hline Low & 12 & 4 & 8 & 0.089 & 6 & 6 & 0.820 & 8 & 4 & 0.160 \\
\hline Moderate & 79 & 47 & 32 & & 43 & 36 & & 51 & 28 & \\
\hline High & 7 & 2 & 5 & & 4 & 3 & & 7 & 0 & \\
\hline \multicolumn{11}{|l|}{$\mathrm{T}$ grade } \\
\hline $\mathrm{T} 1$ & 4 & 1 & 3 & 0.155 & 4 & 0 & 0.060 & 3 & 1 & 0.160 \\
\hline $\mathrm{T} 2$ & 7 & 4 & 3 & & 3 & 4 & & 2 & 5 & \\
\hline T3 & 84 & 48 & 36 & & 46 & 38 & & 59 & 25 & \\
\hline Absent & 81 & 44 & 37 & 1.000 & 43 & 38 & 0.790 & 53 & 28 & 0.570 \\
\hline Present & 17 & 9 & 8 & & 10 & 7 & & 13 & 4 & \\
\hline \multicolumn{11}{|l|}{ Dukes' stage } \\
\hline A & 8 & 3 & 5 & 0.117 & 6 & 2 & 0.310 & 4 & 4 & 0.060 \\
\hline B & 43 & 25 & 18 & & 25 & 18 & & 24 & 19 & \\
\hline C & 31 & 20 & 11 & & 13 & 18 & & 25 & 6 & \\
\hline D & 16 & 5 & 11 & & 9 & 7 & & 13 & 3 & \\
\hline \multicolumn{11}{|c|}{ Mucinous adenocarcinoma } \\
\hline No & 88 & 49 & 39 & 0.505 & 47 & 41 & 0.750 & 56 & 32 & $0.030^{\star}$ \\
\hline Yes & 10 & 4 & 6 & & 6 & 4 & & 10 & 0 & \\
\hline
\end{tabular}

*, significantly different by the Chi-squared test. 

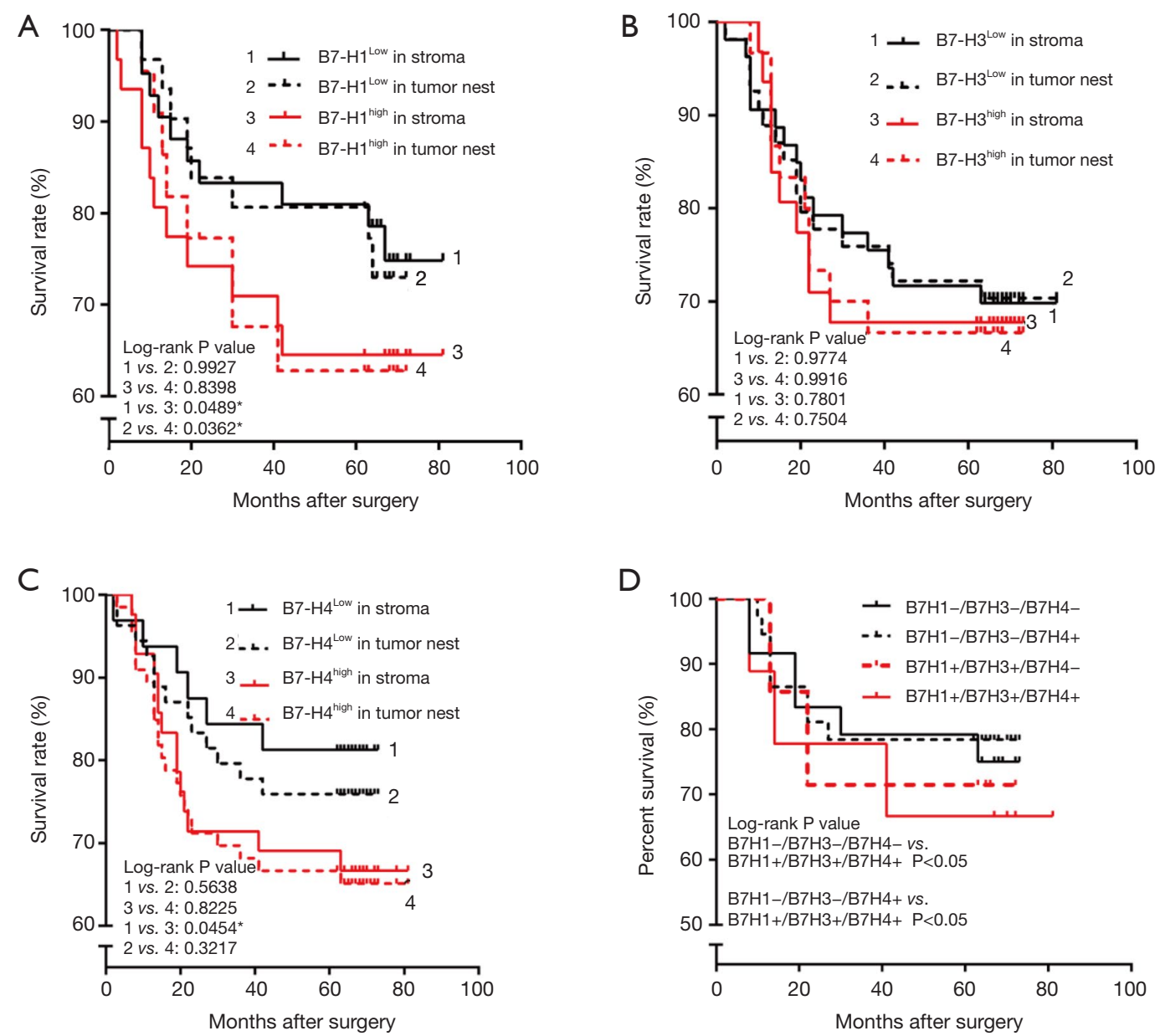

Figure 3 The relationship between the co-expression of B7-H1, B7-H3, and B7-H4 molecules and prognosis of patients with colorectal cancer. They were grouped according to the level of B7-H1 expression in the tumor foci and stroma of colorectal cancer. The results showed that the prognosis of B7-H1 low expression was significantly better than high expression (A). Grouping according to the level of B7-H3 expression, the results showed no significant correlation (B). According to the high and low grouping of B7-H4 expression in the stroma of colorectal cancer, the prognosis of low expression of B7-H4 is significantly better than that of high expression (C). All patients with high expression of B7-H1, B7-H3, B7-H4 have a worse prognosis than those with low expression (D). *, $\mathrm{P}<0.05$.

carcinoma. B7-H4 molecules might take a part in the most important immunosuppressive effect of the immune escape phase.

\section{The relationship between the co-expression of $B 7$ family molecules and prognosis of patients with CRC}

We performed a seven-year survival analysis using the Kaplan-Meier method and the log-rank test. In 98 CRC patients, the survival rate of patients with low B7-H1 expression in the tumor stroma and the foci was higher than that of the patients with high B7-H1 expression. This confirms that $\mathrm{B} 7-\mathrm{H} 1$ is an independent prognostic factor for CRC (Figure 3A). There was no significant correlation between survival time and B7-H3 expression in tumor stroma and tumor foci (Figure $3 B$ ). Expression of B7-H4 in the tumor stroma had higher a survival rate in patients with low $\mathrm{B} 7-\mathrm{H} 4$ expression than those with high $\mathrm{B} 7-\mathrm{H} 4$ expression $(\mathrm{P}=0.045)$, but there was no significant correlation observed in cancer foci (Figure 3C). Furthermore, analysis of co-expression of B7 family molecules and prognosis in $98 \mathrm{CRC}$ patients found that $92.9 \%$ of CRC patients expressed B7 negative costimulatory molecules in different degrees. The prognosis 
A
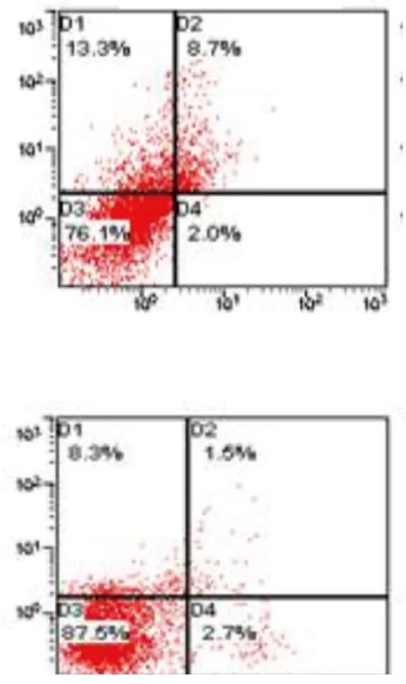

B

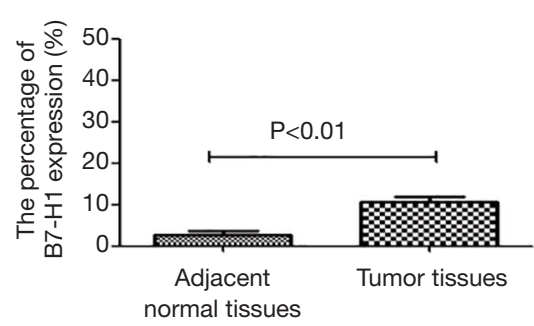

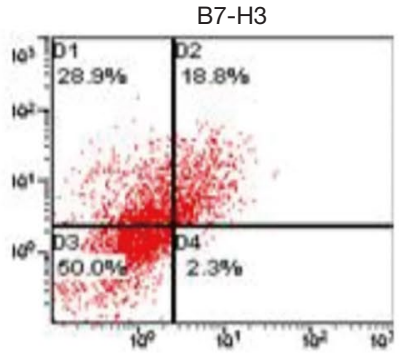

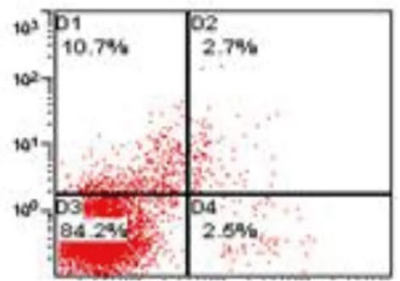

B7- 44

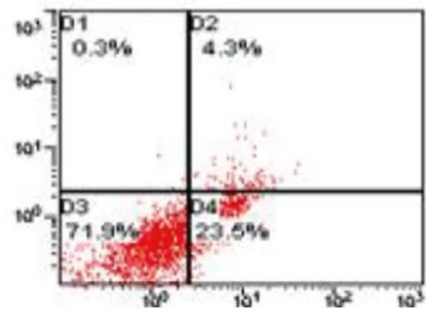

Tumor

tissue

$\mathrm{CD}^{+} \mathrm{T}$ cell

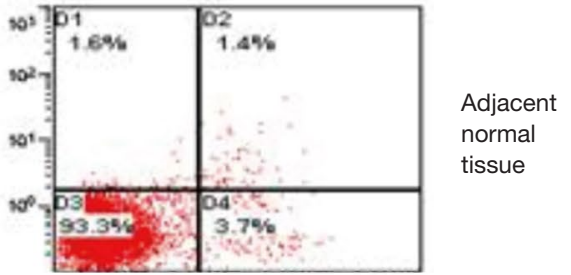

Figure 4 The relationship between $\mathrm{CD}^{+} \mathrm{T}$ cell infiltration and co-expression of B7-H1, B7-H3, and B7-H4 in colorectal cancer. The expression of $\mathrm{B} 7-\mathrm{H} 1, \mathrm{~B} 7-\mathrm{H} 3$ and $\mathrm{B} 7-\mathrm{H} 4$ in $\mathrm{CD} 3^{+}$lymphocytes of colorectal cancer tissue was significantly higher than that in adjacent normal tissues (A). Colorectal cancer tissue and normal tissue $\mathrm{CD}^{+}$lymphocyte expression B7-H1, B7-H3, B7-H4 (P<0.05 for statistical difference) (B).

was negatively correlated with the number of co-expressing B7 negative costimulatory molecules (Figure 3D).

\section{The relationship between $\mathrm{CD}^{+} \mathrm{T}$ cell infiltration and co-expression of B7-H1, B7-H3, and B7-H4 in CRC}

We used flow cytometry analysis to measure the expression of B7 family molecules in $\mathrm{CD}^{+}$lymphocytes in colorectal carcinoma tissues and adjacent normal tissues (Figure 4A). The expression of its was significantly higher in $\mathrm{CD}^{+}$ lymphocytes in colorectal carcinoma tissue than in adjacent normal tissues, and this difference was statistically significant (Figure 4B).

\section{Discussion}

The three molecules of B7 family, play a negative costimulatory effect during the carcinogenesis, and they negatively regulate the immune response by inhibiting the activation and proliferation of $\mathrm{T}$ cells (20). They were closely related to the immune escape mechanism of tumor cells, tumor proliferation, invasion, and metastasis. In the development of CRC, there were differences between the expression of these three negative co-stimulatory molecules in the benign and malignant lesions. The expression of $\mathrm{B} 7-\mathrm{H} 1$ and $\mathrm{B} 7-\mathrm{H} 3$ proteins were upregulated in colorectal polyps, whereas B7-H4 was only expressed during cancerous stages. The expression level of $\mathrm{B} 7-\mathrm{H} 4$ in colorectal tumorous interstitial lymphocytes was related to patient age, lymph node metastasis, and whether or not it was a mucinous adenocarcinoma (21). The expression level of B7-H4 in cancer foci was related to tumor stage, distant metastasis, lymph node metastasis, and Dukes' staging. Survival analysis showed that the expression of B7-H1 
and $\mathrm{B} 7-\mathrm{H} 4$ molecules in colorectal carcinoma was related to the survival rate of colorectal carcinoma patients, but there was no statistically significant correlation between the expression of $\mathrm{B} 7-\mathrm{H} 3$ and the survival rate of CRC patients. Our analysis of the co-expression of B7 family molecules in CRC and the prognosis of patients found that 92.9\% of CRC patients expressed the B7 family of negative costimulatory molecules in different degrees. We also found that the prognosis is negatively correlated with the number of co-expressions of the negative costimulatory molecules in the B7 family. The correlation between these three negative co-stimulatory molecular expression levels with tumor pathological data and patient survival time has important clinical application value for clinical judgment of patient prognosis and guiding postoperative treatment.

B7 family molecules demonstrated a high degree of similarity in both mRNA and protein expression (22). In particular, mRNA was all expressed in various nonlymphoid tissues, including intestine, stomach, and lung. Investigation of the expression of B7 family molecules in various stages of $\mathrm{CRC}$ revealed that the $\mathrm{B} 7$ family negative molecules were barely expressed in normal colorectal tissue. The expression of B7 family negative molecules B7-H1 and $\mathrm{B} 7-\mathrm{H} 3$ began to increase in polyps and continued in the stages of adenoma, high-grade neoplasia, and CRC, but B7-H4 molecules were only expressed in the colorectal carcinoma stage. This suggests that $\mathrm{B} 7-\mathrm{H} 1$ and $\mathrm{B} 7-\mathrm{H} 3$ molecules could be potential warning molecules involved in initiating immune escape and causing CRC $(8,21,23)$. These two molecules continue to be expressed as the tumor progresses, but the $\mathrm{B} 7-\mathrm{H} 4$ molecule only exerted negative regulation during the tumor immune escape phase (24). Therefore, $\mathrm{B} 7-\mathrm{H} 1$ and $\mathrm{B} 7-\mathrm{H} 3$ molecules might be potential targets for the early diagnosis and prevention of tumors, and the intervention of $\mathrm{B} 7-\mathrm{H} 4$ molecules is more likely to provide a new strategy for biological targeted therapy in CRC $(22,25)$.

The expression of $\mathrm{B} 7-\mathrm{H} 1, \mathrm{~B} 7-\mathrm{H} 3$, and $\mathrm{B} 7-\mathrm{H} 4$ molecules on the surface of infiltrating $\mathrm{CD}^{+} \mathrm{T}$ cells in colorectal carcinoma tissues was higher than that in adjacent normal tissues. This suggests that B7-H1, B7$\mathrm{H} 3$, and $\mathrm{B} 7-\mathrm{H} 4$ molecules participate in immune escape. However, when the number of anti-tumor immune cells is sufficient, they can still control tumor progression, which also provides a favorable theoretical explanation for the treatment of adoptive immune cells (26). Further studies on the molecular mechanisms of the B7 family negative molecules could help us to understand the occurrence and development of colorectal carcinoma, and it may provide new intervention strategies for the early diagnosis and targeted treatment of colorectal carcinoma.

\section{Acknowledgments}

Funding: This study was supported by grants from the National Natural Science Foundation Youth Project of China (No. 81502042), the grant from the Natural Science Foundation of Jiangsu Province (No. BK20171150).

\section{Footnote}

Conflicts of Interest: All authors have completed the ICMJE uniform disclosure form (available at http://dx.doi. org/10.21037/tcr.2018.07.15). The authors have no conflicts of interest to declare.

Ethical Statement: The authors are accountable for all aspects of the work in ensuring that questions related to the accuracy or integrity of any part of the work are appropriately investigated and resolved. The study was conducted in accordance with the Declaration of Helsinki (as revised in 2013). Ethical Review Committee of the Affiliated Hospital of Jiangnan University approved the experimental protocols. Informed consent was obtained from all patients.

Open Access Statement: This is an Open Access article distributed in accordance with the Creative Commons Attribution-NonCommercial-NoDerivs 4.0 International License (CC BY-NC-ND 4.0), which permits the noncommercial replication and distribution of the article with the strict proviso that no changes or edits are made and the original work is properly cited (including links to both the formal publication through the relevant DOI and the license). See: https://creativecommons.org/licenses/by-nc-nd/4.0/.

\section{References}

1. Ilyas M, Straub J, Tomlinson IP, et al. Genetic pathways in colorectal and other cancers. Eur J Cancer 1999;35:1986-2002.

2. Stutman O. Immunodepression and malignancy. Adv Cancer Res 1975;22:261-422.

3. Pardoll D. Does the immune system see tumors as foreign or self? Annu Rev Immunol 2003;21:807-39.

4. Kaplan DH, Shankaran V, Dighe AS, et al. Demonstration of an interferon gamma-dependent tumor surveillance 
system in immunocompetent mice. Proc Natl Acad Sci U S A 1998;95:7556-61.

5. Shi SJ, Ding ML, Wang LJ, et al. CD4+T cell specific B7$\mathrm{H} 1$ selectively inhibits proliferation of negative $\mathrm{T}$ cells and Th17 differentiation in experimental autoimmune encephalomyelitis. Oncotarget 2017;8:90028-36.

6. Zhi Y, Mou Z, Chen J, et al. B7H1 Expression and Epithelial-To-Mesenchymal Transition Phenotypes on Colorectal Cancer Stem-Like Cells. PLoS One 2015;10:e0135528.

7. Deng R, Cassady K, Li X, et al. B7H1/CD80 interaction augments PD-1-dependent $\mathrm{T}$ cell apoptosis and ameliorates graft versus host disease. J. Immunol 2015;194:560-74.

8. Ding Q, Lu L, Wang B, et al. B7H1-Ig fusion protein activates the CD4+ IFN-gamma receptor+ type $1 \mathrm{~T}$ regulatory subset through IFN-gamma-secreting Th1 cells. J Immunol 2006;177:3606-14.

9. Kim JJ, Xu H, Haddad H, et al. B7H1 expression and association with clinical response to sunitinib therapy in patients with metastatic renal cell carcinoma (mRCC). BJU International 2013;103:7.

10. Shanzhi W, Yiping H, Ling H, et al. The relationship between TTF-1 expression and EGFR mutations in lung adenocarcinomas. PLoS One 2014;9:e95479.

11. Li Y, Huang J, Foley NM, et al. B7H3 ameliorates LPSinduced acute lung injury via attenuation of neutrophil migration and infiltration. Sci Rep 2016;6:31284.

12. Xu F, Yi J, Wang F, et al. Involvement of soluble B7H3 in combination with the serum inflammatory cytokines interleukin 17, 8 and 6 in the diagnosis of hepatocellular carcinoma. Oncol Lett 2017;14:8138-43.

13. Zhao L, Xie C, Liu D, et al. Early Detection of Hepatocellular Carcinoma in Patients with Hepatocirrhosis by Soluble B7-H3. J Gastrointest Surg 2017;21:807-12.

14. Inamura K, Yokouchi Y, Kobayashi M, et al. WITHDRAWN: Tumor B7-H3 (CD276) expression and smoking history in relation to lung adenocarcinoma prognosis. Lung Cancer 2016;243:21-8.

15. Li Y, Guo G, Jie S, et al. B7-H3 Promotes the Migration and Invasion of Human Bladder Cancer Cells via the PI3K/Akt/STAT3 Signaling Pathway. J Cancer 2017;8:816-24.

16. Kasten BB, Arend R, Katre A, et al. B7-H3-targeted (212) $\mathrm{Pb}$ radioimmunotherapy of ovarian cancer in preclinical models. Nucl. Med. Biol 2017;47:23-30.
17. Zhang $\mathrm{P}$, Zhen $\mathrm{C}$, Ning $\mathrm{K}$, et al. Inhibition of $\mathrm{B} 7-\mathrm{H} 3$ reverses oxaliplatin resistance in human colorectal cancer cells. Biochem Biophys Res Commun 2017;490:1132-8.

18. Mao Y, Chen L, Wang F, et al. Cancer cell-expressed B7-H3 regulates the differentiation of tumor-associated macrophages in human colorectal carcinoma. Oncol Lett 2017;14:6177-83.

19. Yang ZZ, Li L, Xu M, et al. Brain-derived neurotrophic factor involved epigenetic repression of UGT2B7 in colorectal carcinoma: A mechanism to alter morphine glucuronidation in tumor. Oncotarget 2017;8:29138-50.

20. Liang M, Li J, Wang D, et al. T-cell infiltration and expressions of T lymphocyte co-inhibitory B7-H1 and B7-H4 molecules among colorectal cancer patients in northeast China's Heilongjiang province. Tumour Biol 2014;35:55-60.

21. Zhou X, Mao Y, Zhu J, et al. TGF- $\beta 1$ promotes colorectal cancer immune escape by elevating $\mathrm{B} 7-\mathrm{H} 3$ and $\mathrm{B} 7-\mathrm{H} 4$ via the miR-155/miR-143 axis. Oncotarget 2016;7:67196-211.

22. Peng $\mathrm{HX}, \mathrm{Wu} W$, Yang D, et al. Role of B7-H4 siRNA in Proliferation, Migration, and Invasion of LOVO Colorectal Carcinoma Cell Line. Biomed Res Int 2015;2015:326981.

23. Yao Y, Ye H, Qi Z, et al. B7-H4(B7x)-Mediated Crosstalk between Glioma-Initiating Cells and Macrophages via the IL6/JAK/STAT3 Pathway Lead to Poor Prognosis in Glioma Patients. Clin Cancer Res 2016;22:2778.

24. Zhao H, Cheng Y, Dong S, et al. Down Regulation of miR-143 Promotes Radiation - Induced Thymic Lymphoma by Targeting B7H1. Toxicol Lett 2017;280:116-24.

25. Lee $\mathrm{YH}$, Martin-Orozco $\mathrm{N}$, Zheng $\mathrm{P}$, et al. Inhibition of the B7-H3 immune checkpoint limits tumor growth by enhancing cytotoxic lymphocyte function. Cell Res 2017;27:1034-45.

26. Seaman S, Zhu Z, Saha S, et al. Eradication of Tumors through Simultaneous Ablation of CD276/B7-H3-Positive Tumor Cells and Tumor Vasculature. Cancer Cell 2017;31:501-15.e8.

Cite this article as: Qiu Z, Wu J, Wang Y, Wang F, Ge X, Liu X, Qi X, Hua D, Mao Y. Expression and clinical significance of negative costimulatory molecules B7-H1, B7-H3 and B7-H4 in the process of colorectal cancer's evolution. Transl Cancer Res 2018;7(4):1026-1035. doi: 10.21037/tcr.2018.07.15 